\title{
Proceedings of the 2009 annual meeting of the Fetal Alcohol Spectrum Disorders Study Group
}

\author{
Feng C. Zhou a, ${ }^{\star}$, Cynthia J.M. Kane ${ }^{b}$, and Susan M. Smith ${ }^{c}$ \\ aDepartment of Anatomy and Cell Biology, Indiana University School of Medicine, Indianapolis, \\ IN, USA \\ bepartment of Neurobiology and Developmental Sciences, University of Arkansas for Medical \\ Sciences, Little Rock, AR, USA \\ 'Department of Nutritional Sciences, University of Wisconsin-Madison, Madison, WI, USA
}

\begin{abstract}
The annual meeting of the Fetal Alcohol Spectrum Disorders Study Group (FASDSG) was held on June 20, 2009 in San Diego, CA, as a satellite of the Research Society on Alcoholism Meeting. The FASDSG membership includes clinical, basic, and social scientists who meet to discuss recent advances and issues in Fetal Alcohol Spectrum Disorders research. The main theme of the meeting was "Epigenetics and Development." Two keynote speakers, Dr. Randy Jirtle and Dr. Michael Skinner, addressed the role of epigenetics and environmental inputs, including alcohol, during critical stages of development and their potential critical and long-lasting effects. Members of the FASDSG provided new findings through brief "FASt" data reports, and national agency representatives provided updates on activities and funding priorities. Scientific presentations were made by recipients of the Student Research Merit Award and Rosett Award.
\end{abstract}

\section{Keywords}

Fetal alcohol syndrome; Fetal development; Organization on fetal alcohol spectrum disorders; Epigenetics; Alcoholism; FASDSG

The 2009 Fetal Alcohol Spectrum Disorders Study Group (FASDSG) met on June 20, 2009, in San Diego, CA, as a satellite of the Research Society on Alcoholism Meeting. The 20082009 FASDSG officers, Feng Zhou (President), Cindy Kane (Vice-President), and Susan Smith (Secretary-Treasurer) organized the meeting. The FASDSG membership includes clinical, basic, and social scientists who meet to discuss recent advances and issues in fetal alcohol spectrum disorders (FASD) research. More than 161 individuals attended the study group, including more than 100 FASDSG professionals and approximately 60 students. Attendees were largely from the United States and included researchers from Canada, South Africa, Norway, Spain, Brazil, Russia, New Zealand, and Israel. In this meeting, a new logo (Fig. 1, right panel) was adopted to represent FASDSG, and a Web page for the Study Group was introduced in the beginning of the meeting (www.FASDSG.org; Fig. 1). 


\section{Keynote speech}

The main theme of the meeting was "Epigenetics and Development." There is growing awareness that gestational alcohol exposure might alter fetal epigenetic programming, potentially affecting both developmental sensitivity to alcohol and gestational outcome. The first keynote speaker was Dr. Randy Jirtle, PhD, Professor and Director of the Epigenetics and Imprinting Laboratory at Duke University Medical Center. The title of the presentation was "Epigenetics and the fetal origins of disease susceptibility." In linking environmental input, epigenetics, and developmental disorder, Dr. Jirtle first posed the questions: "Why do we vary in our susceptibility to diseases?" And, "why do identical twins vary in their susceptibility to diseases and disorders if the only thing that really matters is the genetic variation?" Dr. Jirtle pointed out that slight variations in the environment in the womb can create susceptibility variations later in life (Jirtle and Skinner, 2007). He explained that epigenetics, "above genetics," refers to the study of heritable changes in gene function during cell replication that occur without a change in the DNA sequence itself. The two basic mechanisms associated with epigenetics are DNA methylation and histone modifications. The two together alter chromatin structure. He used an analogy that the genome is comparable to the hardware of a computer, whereas the epigenome is comparable to the software of a computer, that is, telling the computer how, when, and where to work. Two groups of epigenetic genes were introduced: metastable epiallele genes (genes with unusual characteristics of variable expressivity in the absence of genetic heterogeneity) and genomically imprinted genes. He used the Agouti gene model to pose questions about the effects of the womb environment. For example, exposure to dietary supplements was linked to phenotypic and disease susceptibility changes in adulthood and offspring through the mechanism of DNA methylation rather than genomic mutations. This indicates the prominence of nurture over nature. Furthermore, epigenetically induced changes in disease susceptibility, for example, via food, really are important components in medicine. To some degree, humans might be what their mothers ate while they were in the womb. Addressing the second group of epigenetic genes, Dr. Jirtle said that all genes are equal, but some are more equal than others, meaning those genes that are more equal are genomically imprinted genes because they are expressed from one, paternal or maternal, allele. Misrepresentation of DNA methylation on these genes from parental allele may alter gene expression and subsequently introduce disease. He explained theories of how and why these imprinted genes evolved and how imprinted genes influence gene expression. He cited the intragenomic conflict theory of evolutionary biologist, David Haig. This theory posits that genomic imprinting and parent-offspring conflict are remnants of a genetic battle between male and female parents to control nutrient utilization and the growth of offspring (Haig, 1997). An animal model of imprinted genes demonstrated differentially methylated imprinted regulatory elements affecting expression of the genes. He concluded his presentation discussing the characteristics of epigenetic involvement, including imprinted genes and their linkage to diseases, such as cancer and developmental syndromes.

The second keynote speaker was Michael Skinner, PhD, Professor at the Center for Reproductive Biology at the School of Molecular Biosciences at Washington State University. The title of the presentation was "Epigenetic transgenerational actions of endocrine disruptors on reproduction and disease: The ghosts in your genes." Dr. Skinner began by explaining how epigenetics play a role in environmentally mediated adult onset diseases and in transgenerational phenomena. He stressed that the individual is exposed to the environmental factor, but so too is the subsequent progeny. For example, in his published work, the environmental compounds, methoxychlor (a pesticide and replacement for DDT) and vinclozolin (a fungicide), can bind to hormone receptors and are pathogenic through more than one generation (Anway et al., 2005, 2006). His research identified a paternal allele that was transgenerational or permanently reprogrammed in subsequent 
generations. Transgenerational effects of environmental toxicants (e.g., endocrine disruptors) significantly amplified the impact and health hazards of these compounds. One of the most sensitive periods to exposure is during embryonic gonadal sex determination when the germ line is undergoing epigenetic programming and DNA remethylation. Endocrine disruptors can affect embryonic testis development and subsequently cause an increase in spermatogenic cell apoptosis in the adult. Interestingly, this spermatogenic defect is transgenerational (F1, F2, F3, and F4 generations) and hypothesized to be because of a permanent alteration of DNA methylation in the germ line. This appears to involve the induction of new imprinted DNA methylation sites that regulate transcription distally. Regions of differential DNA methylation were identified with chromatin immunoprecipitation-Chip analysis in F3 generation sperm. The expression of more than 200 genes was altered in the embryonic testis, and surprisingly, this altered transcriptome was similar for all generations (F1-F3). All tissues that were examined had a transgenerational transcriptome effect that was tissue specific. In addition to the detection of the male testis disorder, transgenerational effects on other disease states were observed as the animals aged, including tumor development, prostate disease, kidney disease, and immune abnormalities. Recent observations suggest transgenerational effects on behaviors, such as sexual selection and anxiety. Therefore, the transgenerational epigenetic mechanism appears to involve the actions of an environmental compound at the time of sex determination. This, in turn, alters the epigenetic (i.e., DNA methylation) programming of the germ line permanently and alters the transcriptomes of developing organs to induce disease development transgenerationally (Skinner et al., 2008). Dr. Skinner concluded with the suggestion that environmental factors can reprogram the germ line to induce epigenetic transgenerational disease and is a new paradigm in disease etiology.

\section{"FASt" data presentation}

As in past years, members of the FASDSG gave presentations during "FASt" data sessions, which provided brief updates on research findings. Each 5-min presentation consisted of a single slide followed with 1 min of questions and answers. A total of 16 talks were given in three sessions that focused on pathogenesis: new approaches and new horizons; clinical and translational research; and function and treatment. Seven of these presentations were by students and postdoctoral researchers.

"FASt" data presentations I-pathogenesis: new approaches and new horizons

Rola A. Bekdash, N. I. Boyadjieva, and D. K. Sarkar (Rutgers University) demonstrated that prenatal ethanol exposure suppressed the beta-endorphin producing proopiomelanocortin gene by altering histone methylation at specific sites. The demethylating agent, 5-azacytidine, decreased methylation only in the absence but not in the presence of ethanol.

Conny Lin, Y. Li, and C. H. Rankin (University of British Columbia) found different levels of growth retardation in a genetically identical nematode model, Caenorhabditis elegans exposed to the same embryonic ethanol exposure condition. This phenotypic variation may be caused by differences in epigenetically mediated alteration in gene expression in response to ethanol exposure.

Steven D. Hicks, F. A Middleton, and M. W. Miller (State University of New York, Upstate Medical University) showed that ethanol can interfere with neural stem cell (NSC) proliferation through DNA damage and hypermethylation that alters the expression of genes involved in cell cycle regulation, which in turn prohibit the progression of NSCs through G1/S and G2/M checkpoints. 
Elizabeth A. Godin-Myers, S. K. O'Leary-Moore, A. A. Khan, J. J. Ament, S. E. Parnell, D. B. Dehart, M. A. Styner, G. A. Johnson, K. K. Sulik (University of North Carolina \& Duke University) used magnetic resonance imaging (MRI) to discover cerebral cortical heterotopias/dysplasia in mice exposed to binge alcohol during the week 3-6 human pregnancy equivalent.

Kristina A. Uban, J. H. Sliwowska, S. Leiblich, L. A. M. Galea, and J. Weinberg (University of British Columbia) found that the pattern of stress-induced alteration in neurogenesis in prenatal alcohol-exposed female rats was different than that found in the male rats. Stress induced an increase in cell proliferation in unexposed female rats but not in ethanol-exposed or pair-fed female rats, whereas stress suppressed neurogenesis in male rats. Both neuron and glial cell survival were suppressed in ethanol-exposed females under both stress and nonstressed conditions.

"FASt" data presentations II—clinical and translational research

Tatiana Balachova, L. Tsvetkova (St. Petersburg State University, Russia), and the Prevent Fetal Alcohol Syndrome (FAS) Research Group reported that FASD information brochures with negative vivid images raised higher emotional responses than ones with positive images, and they improved knowledge and attitudes toward drinking during pregnancy. However, they were not effective in reducing alcohol consumption in this population.

Philip A. May (University of New Mexico) reported the prevalence of FAS as 6.7 per 1,000 children and FASD as $2.5 \%$ from two epidemiological studies done in Italy and the United States. The prevalence in South Africa was even higher, at 50.1 per 1,000 for FAS and 7.2\% for FASD. These rates were much higher than the previously accepted estimate of 1-3 per 1,000 for FAS and $1 \%$ for FASD.

Bruce Anthony, S. Vinci-Booher, L. Wetherill, R. Ward, C. Goodlett, and F. C. Zhou (Indiana University School of Medicine) reported that the three-dimensional morpho-metric analysis of facial dysmorphology successfully used in human studies could reliably discriminate alcohol-exposed mice embryo from nonexposed embryo. This effort will benefit the understanding of facial dysmorphology associated with various alcohol exposure conditions using the mouse model of FASD.

Leah F. Wetherill, C. P. Klingenberg, J. Rogers, E. Moore, R. Ward, I. Autti-Räamäo, A. Fagerlund, S. W. Jacobson, S. N. Mattson, T. Foroud, and the Collaborative Initiative on Fetal Alcohol Spectrum Disorders consortium (St. Vincent Hospital, Indianapolis) reported the improved algorithm in the computerized anthropometry that is used to identify FAS facial features in different ethnicities. Original facial landmarks were configured into the same size, centered on a single gravity point, and rotated to minimize dispersion. The resulting analysis was excellent in discriminating FAS from control subjects in at least two populations: Cape Town in South Africa and Helsinki in Finland.

Christina D. Chambers, J. Y. Uriu-Adams, C. L. Keen, L. Yevtushok, and W. W. Wertelecki (University of California, San Diego) reported on the nutrient status of alcoholexposed women during midgestation in Ukraine. Pregnant women exposed to moderate to heavy alcohol had lower $\mathrm{Cu}$ and tartrate-resistant acid phosphatase activity.

Julie Kable, C. Coles, M. E. Lynch, and T. Gauthier (Emory University School of Medicine) found that antioxidant glutathione and its oxidized component glutathione disulfide were altered in women who drank during pregnancy. Their 6-month-old infants showed diminished neurophysiological encoding assessed by cardiac orienting responses to speech sounds. 


\section{"FASt" data presentations III-function and treatment}

Roh-Yu Shen, S. Haj-Dahmane, and J. Wang (University at Buffalo) found that ethanol exposure during gestational day 8-20 caused an increase in a-amino-3-hydroxy-5-methyl-4isoxazolepropionic acid receptor excitatory post-synaptic currents and abolishment of glutamate-mediated long-term depression (LTD) in the ventral tegmentum area of 3-6week-old rats. Ethanol exposure caused a reduction in the retrograde endocannabinoid messenger that was responsible for the decrease of glutamate release, which mediated the abolishment of LTD.

Nathen J. Murawski and M. E. Stanton (University of Delaware) demonstrated that rats exposed to ethanol over postnatal day 4-9 were impaired in a variant of the contextual fear conditioning paradigm, the context preexposure facilitation effect (CPEF). As opposed to the standard contextual fear conditioning paradigm, the CPEF was more dependent on hippocampal function, and ethanol-exposed rats showed more reliable impairment in the CPEF.

Crystal L. Lantz and A. E. Medina (Virginia Commonwealth University) examined the effect of ethanol exposure during the third trimester equivalent (P5-9) on ocular dominance plasticity in mice. Ethanol-exposed animals did not exhibit reduced responses in the deprived eye after monocular deprivation was seen in controls. This ethanol-inhibited ocular plasticity was rescued by injecting a phosphodiesterase type I inhibitor (vinpocetine) before the onset of monocular deprivation.

Rafael K. Varaschin, D. A. Hamilton, and D. D. Savage (University of New Mexico) reported that the long-term potentiation (LTP) deficits in dentate gyrus found in rats whose mother was fed a 5\% ethanol liquid diet were also found in rats whose mother was exposed to ethanol for a shorter duration ( $4 \mathrm{~h} / \mathrm{day}, 5 \%$ in water). This LTP deficit could be elicited by a submaximal tentanizing stimulus but not a saturating stimulus, and this impairment could be reversed by ABT-239, a histamine type 3 receptor antagonist, which suggests a histaminergic regulation of LTP in the dentate gyrus of fetal ethanol-exposed rats.

\section{Agency updates}

Representatives from governmental agencies provided reports on news and updates.

Dale Hereld, MD, PhD, National Institute on Alcohol Abuse \& Alcoholism (NIAAA), spoke about the importance of alcohol's effects throughout life from the moment of conception forward. He iterated the need to identify mechanisms underlying FASD and development of preventive measures. He gave an overview of NIAAA's strategic plan, which included enhancing the understanding of FASD etiology, increasing early detection of FASD dysmorphology, advancing case recognition of FASD, developing preventive measures based on an understanding of the neuro-behavioral phenotype, and developing preventive measures by changing social norms. The NIAAA's budget and funding grants in the support of FASD were illustrated; for example, the two research initiatives that he addressed were Collaborative Initiative of FASD and Prenatal Alcohol in Sudden Infant Death Syndrome and Stillbirth Network. The Requests for Applications and a Program Announcement and the stimulus act funding opportunities were further mentioned. $\mathrm{He}$ concluded with a brief overview of NIAAA's publications related to FASD.

Sally M. Anderson, PhD, Interagency Coordinating Committee on FASD (ICCFASD), began with an overview of the ICC, an interagency that works to increase communication, cooperation, and collaboration among the many disciplines and federal agencies that address prenatal alcohol exposure. It is supported and funded by NIAAA. She discussed activities 
under ICCFASD, including the Department of Education's programs, which educate people about FASD, Indian Health Services, Health Resources and Services Administration, and Agency for Healthcare Research and Quality. She described the National Children's Study, a nationwide prospective study that will recruit and collect outcomes data on a large child cohort, spanning the period from prenatally to 20 years of age. She further elaborated on the work groups of ICCFASD, including the Work Group on Diagnostic Issues, and upcoming meetings.

Clark Denny, PhD, Centers for Disease Control (CDC) and Prevention, announced the newly funded research training centers and introduced the new FASD Competency-based Curriculum Development Guide and the National Organization on Fetal Alcohol Syndrome $\mathrm{K}-12$ Curriculum. CDC also funds formative research on alcohol use in pregnancy, which is qualitative research based on 20 focus groups exploring women's views on alcohol use during pregnancy. CDC provides digital stories of FASD and the Prevention Report on Reducing Alcohol-exposed Pregnancies and A Call to Action: Advancing Essential Services and Research on Fetal Alcohol Spectrum Disorders. Denny mentioned the development of Project Changing High-risk AlcOhol Use and Increasing Contraception Effectiveness Study and announced funding opportunities. He concluded with an overview of the Intervening with Children Project.

Callie Gass, Substance Abuse and Mental Health Services Administration (SAMHSA), FASD Center for Excellence, presented an overview of SAMHSA's approach to responding to FASD. She announced the plans of the Native Initiative (over the next 4 years) in Native American communities, which involve forming task forces to gather information to find out what preventive measures work best in the communities. She introduced the map of new participating states on their Web site (http://www.samhsa.gov/) and the new enhanced Spanish-Language site and introduced other subcontractor activities. She reviewed the eight diagnosis and intervention center teams and criteria and reviewed screening children and tracking trends in diagnosis and preventive measures. She concluded with an announcement of forthcoming publications.

\section{Award presentations}

This year, seven young investigators were awarded with travel awards. The 2009 FASDSG Merit Award was presented to graduate student Kelly Nash at the University of Toronto for her presentation on "Voxel-based morphometry of the frontal lobes in children with FASD," studied with Stevens S. A., Skocic J., and Rovet J. Her study was guided by Dr. Joanne Rovet. Nash presented the MRI technique, voxel-based morphometry, to identify the specific gray matter differences in frontal and related areas. Her research differentiated children with FASD from unexposed children, and her work suggested that gray matter in the frontostriatal network important for attention and executive function may be particularly sensitive to prenatal alcohol exposure.

The program's highlight was the presentation of the Rosett Award, a prestigious recognition for important contributions to the field of fetal alcohol research, to Dr. Kathleen K. Sulik, Professor, Bowles Center for Alcohol Studies, University of North Carolina. Sulik received this award for her dedication to research in FAS and her work to educate the public about the disorder. Her many accomplishments include pioneering the development of a mouse FAS model, identifying gastrulation as a target of alcohol, and discovering parallels between FAS and other developmental disorders. Dr. Sulik presented an enlightening overview of her elegant imaging research on early human embryos in different stages of development. 


\section{Acknowledgments}

Funding for this conference was made possible in part by 1R13AA15661-06 from NIAAA. Neither the views expressed in written conference materials or publications and by speakers and moderators necessarily reflect the official policies of the Department of Health and Human Services nor does mention by trade names, commercial practices, or organizations imply endorsement by the US government.

\section{References}

Anway M, Cupp AS, Uzumcu M, Skinner MK. Epigenetic transgenerational actions of endocrine disruptors and male fertility. Science. 2005; 308:1466-1469. [PubMed: 15933200]

Anway MD, Leathers C, Skinner MK. Endocrine disruptor vinclozolin induced epigenetic transgenerational adult onset disease. Endocrinology. 2006; 147:5515-5523. [PubMed: 16973726]

Haig D. Parental antagonism, relatedness asymmetries, and genomic imprinting. Proc. Biol. Sci. 1997; 264:1657-1662. [PubMed: 9404029]

Jirtle RL, Skinner MK. Environmental epigenomics and disease susceptibility. Nat. Rev. Genet. 2007; 8:253-262. [PubMed: 17363974]

Skinner MK, Anway MD, Savenkova MI, Gore AC, Crews D. Transgenerational epigenetic programming of the brain transcriptome and anxiety behavior: environment-genome interactions. PLoS One. 2008; 3:e3745. [PubMed: 19015723] 


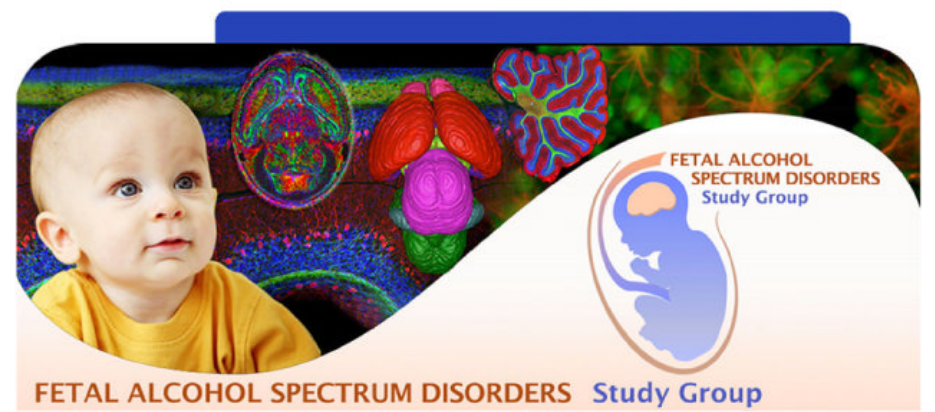

Fig. 1.

The web page of FASDSG "www.FASDSG.org". The logo is shown in the lower right panel. 